\title{
Preliminary Experience of Lvis Blue in the Internal Carotid Artery for The Treatment of Wide-Necked Intracranial Aneurysms
}

\author{
Jeong Hwa Kim ${ }^{1}$, Chang Ki Jang ${ }^{1}$, Jae Whan Lee ${ }^{1}$, Keun Young Park ${ }^{1}$, Joonho Chung ${ }^{1,2}$ \\ ${ }^{1}$ Department of Neurosurgery, Severance Hospital, Yonsei University College of Medicine, Seoul, Korea \\ ${ }^{2}$ Severance Institute for Vascular and Metabolic Research, Yonsei University College of Medicine, Seoul, Korea
}

Received: August 8, 2019

Accepted: August 23, 2019

\section{Corresponding Author:}

Joonho Chung, M.D., Ph.D.

Department of Neurosurgery, Stroke Center, Severance Hospital Severance Institute for Vascular and Metabolic Research Yonsei University College of Medicine 50-1, Yonsei-ro, Seodaemun-gu, Seoul, 03722, Korea

Tel: +82-2-2228-2150

Fax: +82-2-393-9979

E-mail: ns.joonho.chung@gmail. com

\section{Objective}

A new Low-profile Visualized Intraluminal Support device (LVIS Blue) is a braided stent that provides a higher degree of metal coverage and increasingly used for the stent-assisted coiling of intracranial aneurysms. In the present study, our preliminary experience of using LVIS Blue in the internal carotid artery (ICA) for the treatment of wide-necked intracranial aneurysms was reported.

\section{Methods}

This retrospective review included patients with ICA aneurysms prospectively collected from our database who were treated using LVIS Blue at our institution from September 2017 to December 2018. Angiographic results, clinical outcomes, and technical issues were evaluated.

\section{Results}

A total of 22 patients with unruptured wide-necked ICA aneurysms were treated using LVIS Blue. Seven aneurysms were located in the superior hypophyseal artery, 4 in posterior communicating artery, 4 in ICA dorsal wall, 3 in ICA bifurcation, 2 in ophthalmic artery, 1 in ICA ventral wall, and 1 in ICA lateral wall. During the procedure, suboptimal positioning and foreshortening of the stent occurred in 1 patient each. Immediate postembolization angiography showed complete obliteration in 13 patients (59.1\%), residual neck in 4 (18.2\%), and residual aneurysm in 5 patients $(22.7 \%)$. Follow-up angiographic results showed complete obliteration in 19 patients (86.4\%). In-stent stenosis (> 50\% stenosis) was not observed on follow-up angiography. In addition, morbidity or mortality did not occur.

\section{Conclusion}

The LVIS Blue may be feasible and safe for stent-assisted coiling in ICA aneurysms. Foreshortening, suboptimal deployment, and difficulty to open proximal ends may be considerable technical issues in the tortuous ICA.

Keywords: Intracranial aneurysm; Wide-necked aneurysm; LVIS Blue; Stent-assisted coiling 


\section{INTRODUCTION}

Stent-assisted coil embolization (SAC) has been accepted as a feasible option for occluding the wide-necked complex aneurysms with preservation of the parent artery, associated with flow remodeling which promote the complete thrombosis of the coiled aneurysm $^{3,5,12,15)}$. The Low-profile Visualized Intraluminal Support device (LVIS; MicroVention, Inc., Tustin, CA) is a self-expanding, compatible, and widely used closed cell-designed stent. The small cell structure $(0.8 \mathrm{~mm})$ and high metal coverage (approximately $23 \%$ ) allow the achievement of greater complete or near-complete occlusion rate of intracranial aneurysm compared with the currently used microstents ${ }^{8)}$. The LVIS Blue, a new modification of LVIS, is a braided stent that provides a higher degree of metal coverage (approximately 28\%) and is increasingly used for SAC. The LVIS Blue showed improved wall apposition and aneurysm neck coverage ${ }^{11)}$ and has a good crossing profile for microcatheters, better wall apposition and less perforator coverage than a flow redirection endoluminal device (FRED $)^{14)}$.

However, clinical data regarding the safety and effectiveness of LVIS Blue are insufficient. Thus, in the present study, preliminary experience of using LVIS Blue in the internal carotid artery (ICA) for the treatment of wide-necked intracranial aneurysms was reported.

\section{METHODS}

\section{Patient Selection}

This retrospective study was approved by our Institutional Review Board, and the requirement for informed consent was waived. Endovascular treatment of 197 aneurysms (176 unruptured and 21 ruptured) in 129 patients was performed from September 2017 to December 2018. Among 114 aneurysms treated with SAC, 22 cases (all wide-necked unruptured aneurysms in the ICA) were treated with LVIS Blue stents.

All 22 patients underwent digital subtraction angiography (DSA) and rotational angiography (Philips Allura FD20 Clarity System, Philips Medical Systems, Best, The Netherlands) with three-dimensional reconstructions to characterize aneurysm and parent artery anatomy (Allura 3D-RA workstation, Philips Medical Systems). Based on DSA results, the treatment decision was made by consensus among microvascular neurosurgeons and neurointerventionists. Wide-necked aneurysms were defined as having a neck width $\geq 4 \mathrm{~mm}$ or a dome-to-neck ratio $<2$.

\section{Endovascular Treatment}

All procedures were performed under general anesthesia with a standard approach from the common femoral artery. A 6-Fr or 7-Fr guiding catheter was positioned in the ICA. Intravenous systemic heparin $(50 \mathrm{U} / \mathrm{kg})$ was administered while placing the guiding catheter. An activated clotting time was maintained at 2-2.5 times that of baseline during endovascular treatment. A Headway-21 (MicroVention) or a Prowler Select Plus (Codman Neurovascular) were used to deliver the LVIS Blue stent. The jailing or semi-jailing techniques were primarily performed, and if failed, the through the strut (cell-through) technique was implemented.

Patients took a daily dose of $75 \mathrm{mg}$ of clopidogrel and $100 \mathrm{mg}$ of aspirin for more than 7 days before the SAC procedure. For patients who had insufficient premedication or when the SAC was planned immediately after the diagnostic DSA, loading dose of aspirin and clopidogrel $300 \mathrm{mg}$ each was administered the day before treatment. Platelet function testing was routinely performed for all patients. After the procedure, patients were prescribed 75 $\mathrm{mg}$ of clopidogrel daily for 3 months and $100 \mathrm{mg}$ of aspirin daily for at least 12 months.

\section{Clinical and Radiographic Outcomes}

Angiographic and clinical outcomes were retrospectively reviewed. Follow-up angiography was performed in all patients between 6 and 24 months (mean 13.4 months) after the procedure, and clinical follow-up was performed for $6-24$ months (mean 16.5 months). The angiographic results were evaluated by 2 independent investigators and categorized according to the modified Raymond-Roy classification (Class I: complete obliteration, Class II: residual neck, Class IIIa: residual aneurysm, contrast within coil interstices, Class IIIb: residual aneurysm, contrast along aneurysm wall). Clinical outcomes were assessed using the modified Rankin Scale (mRS) and evaluated at the time of discharge and out-patient follow-up by an independent investigator who was not involved in patient care. In-stent stenosis was defined as $>50 \%$ stenosis on follow-up angiography. After the procedure, skull X-ray was performed and diffusion magnetic resonance imaging (diffusion MRI) was conducted at postoperative day 1 . Procedural complication was defined as acute infarct or hemorrhage that impaired the clinical outcome and required prolonged hospital care.

\section{RESULTS}

Table 1 shows the data and outcomes of the 22 patients ( 5 males and 17 females, age range 37 - 77 years, mean 54.1 years). No patient had a history of subarachnoid hemorrhage. All aneurysms were located at the intracranial portion of the ICA with wide-necked saccular type morphology. The most common site 
Table 1. Baseline characteristics and outcomes of 22 patients with 22 aneurysms in the internal carotid artery

\begin{tabular}{|c|c|}
\hline & $\mathrm{N}=22$ \\
\hline Age (year, mean $\pm \mathrm{SD}$ ) & $54.1 \pm 11.1$ \\
\hline Female (n, \%) & $17(77.3)$ \\
\hline Hypertension (n, \%) & $9(40.9)$ \\
\hline Diabetes (n, \%) & $5(22.7)$ \\
\hline Dyslipidemia (n, \%) & $3(13.6)$ \\
\hline Smoking (n, \%) & $3(13.6)$ \\
\hline Aneurysm size (mm, mean \pm SD) & $6.24 \pm 2.37$ \\
\hline Neck size (mm, mean \pm SD) & $4.54 \pm 1.56$ \\
\hline \multicolumn{2}{|l|}{ Location $(\mathrm{n}, \%)$} \\
\hline Superior hypophyseal artery & $7(31.8)$ \\
\hline Ophthalmic artery & $2(9.1)$ \\
\hline Dorsal wall & $4(18.2)$ \\
\hline Ventral wall & $1(4.5)$ \\
\hline Lateral wall & $1(4.5)$ \\
\hline Posterior communicating artery & $4(18.2)$ \\
\hline ICA bifurcation & $3(13.6)$ \\
\hline \multicolumn{2}{|c|}{ Technical considerations of stents (n, \%) } \\
\hline Suboptimal positioning & $1(4.5)$ \\
\hline Foreshortening & $1(4.5)$ \\
\hline \multicolumn{2}{|l|}{ Initial angiographic results $(\mathrm{n}, \%)$} \\
\hline Complete obliteration & $13(59.1)$ \\
\hline Residual Neck & $4(18.2)$ \\
\hline Residual Aneurysm & $5(22.7)$ \\
\hline \multicolumn{2}{|l|}{ Follow-up angiographic results (n, \%) } \\
\hline Complete obliteration & $19(86.4)$ \\
\hline Residual Neck & $0(0)$ \\
\hline Residual Aneurysm & $3(3)$ \\
\hline \multicolumn{2}{|l|}{ Clinical outcomes (n, \%) } \\
\hline Morbidity & $0(0)$ \\
\hline Mortality & $0(0)$ \\
\hline
\end{tabular}

ICA: internal carotid artery; SD: standard deviation.

was superior hypophyseal artery $(\mathrm{n}=7)$ followed by posterior communicating artery $(n=4)$, dorsal wall $(n=4)$, ICA bifurcation $(\mathrm{n}=3)$, ophthalmic artery $(\mathrm{n}=2)$, ventral wall $(\mathrm{n}=1)$, and lateral wall $(\mathrm{n}=1)$. Aneurysm size ranged from $4.94-11.31 \mathrm{~mm}$ (mean $6.24 \pm 2.37 \mathrm{~mm}$ ) and neck diameter from $2.86-7.76 \mathrm{~mm}$ (mean $4.54 \pm 1.56 \mathrm{~mm}$ ). Complications related to delivering or deploying the LVIS Blue stent did not occur. However, suboptimal positioning (Fig. 1) and foreshortening (Fig. 2) of the stent occurred in 1 patient each.

At the end of the procedure, parent vessel patency was confirmed with no evidence of associated flow decrease in the covered vessel. Immediate postembolization angiography showed complete obliteration in 13 patients (59.1\%), residual neck in 4 (18.2\%), and residual aneurysm in 5 (22.7\%). Follow-up angio- graphic results showed complete obliteration in 19 patients (86.4\%). All 4 patients with residual necks and 2 patients with residual aneurysms on the immediate postembolization angiography improved and showed complete obliteration on the follow-up angiography. In-stent stenosis ( $>50 \%$ stenosis) was not observed on follow-up angiography. With respect to clinical outcomes, morbidity or mortality did not occur.

\section{DISCUSSION}

In this preliminary study, LVIS Blue was used for the treatment of patients with unruptured, wide-necked saccular aneurysm in the ICA. Angiographic and clinical outcomes showed the LVIS Blue is a feasible and safe device with favorable occlusion rate. Several technical issues related to the mechanical characteristics of the LVIS stent were also elucidated during the procedure, as reported in previous studies.

In recent years, the treatment of intracranial aneurysms focused on the reconstruction of the parent artery and aneurysmal neck. Decrease of intra-aneurysmal flow can be achieved by inserting stents or flow diverters, with or without coil compaction. Stents are believed to redirect the flow in the parent artery and slow down the aneurysmal flow leading to thrombosis and consequently stabilizing the aneurysm ${ }^{11}$. For wide-necked aneurysms, preservation of the parent artery is the technical challenge in the endovascular procedure. The use of self-expandable stents or flow diverters has provided a significant advance in the treatment with high rates of complete occlusion and low rates of recurrence at follow-up ${ }^{9)}$.

The LVIS is a hybrid closed-cell stent made of nickel titanium (nitinol) with flared ends and 2 radiopaque helices of tantalum strands to assist full-length visualization. The device has a high metal-to-surface coverage intended to help promote neo-endothelization. The sliding design of its cells are feasible for crossing the struts with a microcatheter. According to the systematic review of the LVIS device by Zhang et al., the immediate angiographic results in the literature review demonstrated relatively lower rates of complete occlusion, progression to total occlusion was impressive with a high rate of complete occlusion (84.3\%), and the thromboembolic complications were negligible, with a rate of $4.9 \%{ }^{18)}$.

Regarding technical issues, poor wall apposition due to incomplete segmental expansion after application of the LVIS was reported in several studies ${ }^{2,4,7,16}$. Cho et al. reported the poor wall apposition occurred in $9.1 \%$ of patients, with consumption of twist or distortion of LVIS stent at the tortuous segment or acute curve of the $\mathrm{ICA}^{4)}$.

The new low-profile visualized intraluminal support device, 


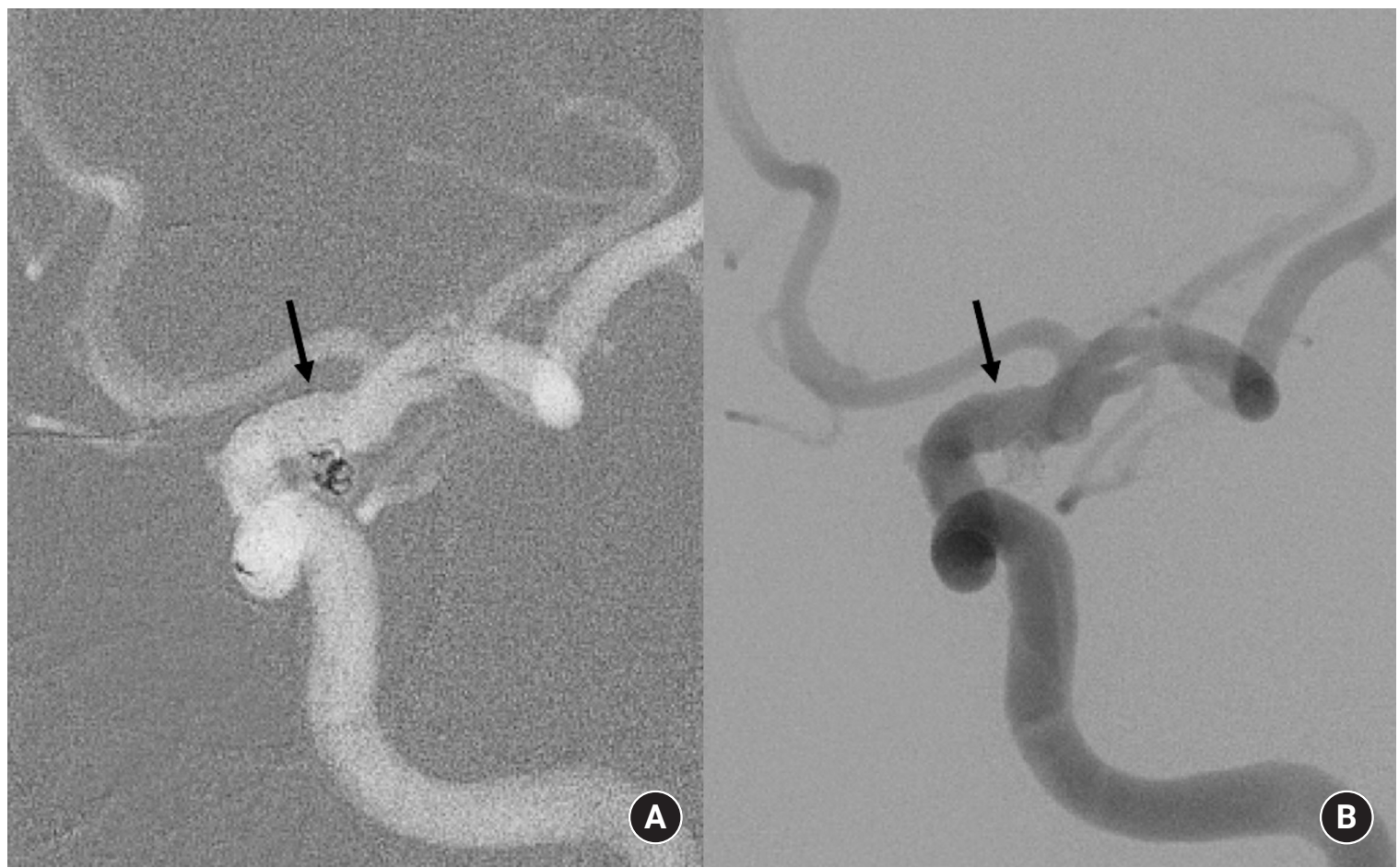

Fig. 1. A 63-year-old female with a left posterior communicating artery aneurysm. The aneurysm is a mitten-shaped saccular sac with a daughter sac. The dome-to-neck ratio is 1.43 with the height of $4.6 \mathrm{~mm}$ and neck size of $3.2 \mathrm{~mm}$. (A) A $4.5 \times 23 \mathrm{~mm}$ LVIS Blue stent was deployed and the distal tips of LVIS Blue stent barely covered the aneurysm neck due to an unstable delivery of microcatheter in the ICA curve. (B) Despite suboptimal positioning of the stent, immediate postembolization and follow-up angiographic results showed complete obliteration of the aneurysm. Black arrows indicate distal tips of the stent.

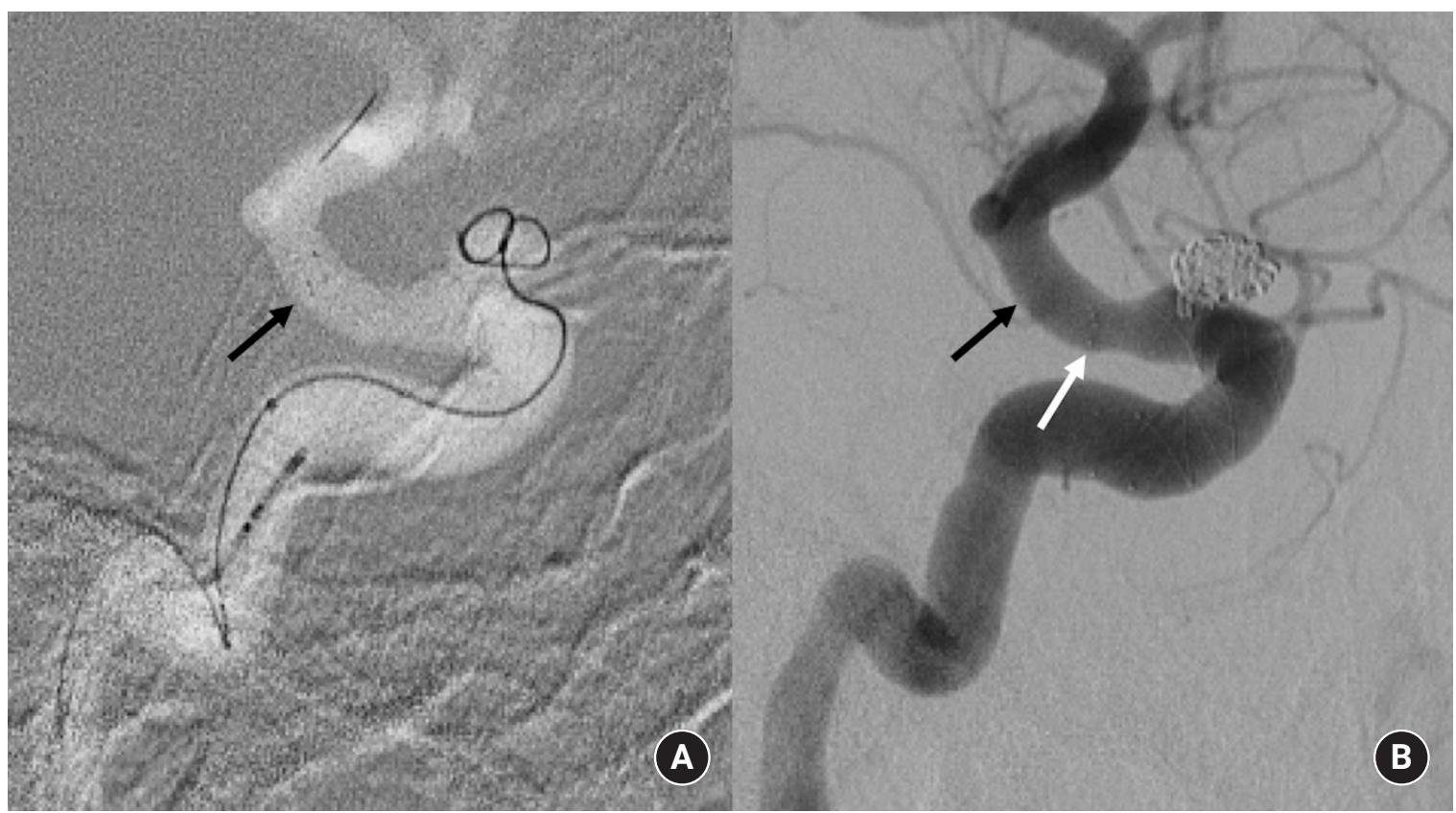

Fig. 2. A 47-year-old male with a dorsal wall aneurysm in the internal carotid artery. The dome-to-neck ratio is 1.35 with a height of $6.9 \mathrm{~mm}$ and neck size of $5.1 \mathrm{~mm}$. (A) A $4.5 \times 23 \mathrm{~mm}$ LVIS Blue was deployed and the jailing technique for coiling was performed. During the procedure, the distal tips of the LVIS Blue showed foreshortening due to unconstrained segment of the aneurysm neck. (B) Immediate postembolization angiography showed residual neck of the aneurysm. Black arrows indicate initial positioning of the stent distal tips. A white arrow indicates final positioning of the stent distal tips after foreshortening. 
LVIS Blue, is a stent with 16 nitinol single wire-braids which is compatible with a 0.021 -inch microcatheter and recommended for a parent vessel $2.5-4.5 \mathrm{~mm}$ in size. The metal coverage of the LVIS Blue is up to $28 \%$, higher than the previous LVIS (approximately 23\%) and lower than the flow redirection endoluminal device (FRED, 30-35\%). In the present study, although immediate angiographic results demonstrated a complete occlusion rate of $59 \%$, progression to total occlusion was impressive with complete obliteration (86.4\%) at the 1-year follow-up; morbidity or mortality did not occur. This result showed better angiographic and clinical outcomes than in previous studies on LVIS or LVIS Blue ${ }^{11,18)}$.

Chung et al. provided informative physical properties of LVIS and LVIS Blue in laboratory studies and suggested the smaller angle of the cell design was modified in LVIS Blue compared with LVIS, leading to a better ability to open the device and greater wall apposition in tortuous arteries and higher aneurysmal neck coverage. The LVIS Blue properties may play an important role in decreasing the development of stent thrombosis and thromboembolism, which occur due to incomplete stent apposition ${ }^{6)}$. In another study, the technical performance based on bench-top and cadaver evaluations was compared between the LVIS Blue and the FRED, several advantages of the LVIS Blue over the FRED were observed. The most important finding was the feasibility for trans-cell approach and lesser perforator coverage with similar inner neck coverage of FRED at the curved vessel models. In addition, the LVIS Blue stent is delivered through lower profile microcatheters (0.021-inch) than the FRED stent (0.027-inch), leading to easier deployment of the stent into more distal vessels and use as an adjunctive method ${ }^{14)}$.

The delayed improvement in aneurysm occlusion after SAC is considered secondary to a 'partial' flow-diverting effect of the modern coil-adjunctive stent ${ }^{17)}$. Jankowitz BT et al. performed a computational flow analysis using 3 aneurysm models, in which Pipeline flow diverter was compared with high porosity (ATLAS, Enterprise) and moderate-to-low porosity (LVIS Blue) stents. The study results demonstrated flow diversion only partially represents a threshold in device design that encompasses metal surface coverage ${ }^{10)}$. This result indicates the LVIS Blue may be beneficial for complete obliteration of an aneurysm due not only to a higher occlusion rate when using coils inside the aneurysm, but also its flow diversion effect.

Several technical issues were elucidated in the present study. In the curved segment of the artery, stent torsion renders opening the proximal tip of the LVIS Blue stent difficult. In addition, the oversized LVIS Blue stent may lead to the development of a transitional zone, where the stent exits the parent artery to the aneurysm neck and the device transitions from a constrained diameter to its unconstrained state, generally resulting in a lower metal coverage surface area. This observation correlated with the previously published results ${ }^{6,14)}$. To overcome this limitation, Lim et al. used an LVIS Blue stent within an Enterprise stent for patients with vertebral artery (VA) dissecting aneurysms, which resulted in successful obliteration while maintaining the VA patency ${ }^{13)}$.

The present study had several limitations including the retrospective data collection and the small number of patients only with an unruptured aneurysm. The follow-up period was short, less than 2-years, and longer follow-up is needed to assess the stability of adequate obliteration. Due to the lack of studies on LVIS Blue stent, comparisons with different devices and laboratory experiments to improve technical defects of LVIS Blue stent should be performed in additional clinical trials.

\section{CONCLUSION}

The LVIS Blue may be feasible and safe for stent-assisted coiling of ICA aneurysms. Foreshortening, suboptimal deployment, and difficulty to open proximal ends may be considerable technical issues in the tortuous ICA. Larger cohort studies with longer follow-up are necessary to support the results from this study.

\section{NOTES}

\section{Conflict of interest}

The authors declare no conflicts of interest.

\section{Ethical approval}

All procedures performed in the studies involving human participants were in accordance with the ethical standards of our Institutional Review Board with the 1964 Helsinki Declaration and its later amendments or comparable ethical standards.

\section{Informed consent}

In this retrospective study, the requirement for informed consent was waived.

\section{Funding}

No funding was received for this study.

\section{REFERENCES}

\footnotetext{
1. Augsburger L, Farhat M, Reymond P, Fonck E, Kulcsar Z, Stergiopulos $\mathrm{N}$, et al. Effect of flow diverter porosity on intraaneurysmal blood flow. Clinical Neuroradiology 2009; 19:204-214.

2. Behme D, Weber A, Kowoll A, Berlis A, Burke TH, Weber W.
} 
Low-profile Visualized Intraluminal Support device (LVIS Jr) as a novel tool in the treatment of wide-necked intracranial aneurysms: initial experience in 32 cases. J Neurointerv Surg 2015;7:281-285.

3. Brinjikji W, Murad MH, Lanzino G, Cloft HJ, Kallmes DF. Endovascular treatment of intracranial aneurysms with flow diverters: a meta-analysis. Stroke 2013;44:442-447.

4. Cho YD, Sohn CH, Kang HS, Kim JE, Cho WS, Hwang G, et al. Coil embolization of intracranial saccular aneurysms using the Low-profile Visualized Intraluminal Support (LVIS) device. Neuroradiology 2014;56:543-551.

5. Chung J, Lim YC, Suh SH, Shim YS, Kim YB, Joo JY, et al. Stent-assisted coil embolization of ruptured wide-necked aneurysms in the acute period: incidence of and risk factors for periprocedural complications. J Neurosurg 2014;121:4-11.

6. Chung J, Matsuda Y, Nelson J, Keigher K, Lopes DK. A new low-profile visualized intraluminal support (LVIS) device, LVIS Blue: laboratory comparison between old and new LVIS. Neurol Res 2018;40:78-85.

7. Fiorella D, Arthur A, Boulos A, Diaz O, Jabbour P, Pride L, et al. Final results of the US humanitarian device exemption study of the low-profile visualized intraluminal support (LVIS) device. J Neurointerv Surg 2016;8:894-897.

8. Ge H, Lv X, Yang X, He H, Jin H, Li Y. LVIS Stent Versus Enterprise Stent for the Treatment of Unruptured Intracranial Aneurysms. World Neurosurg 2016;91:365-370.

9. Geyik S, Yavuz K, Yurttutan N, Saatci I, Cekirge HS. Stent-assisted coiling in endovascular treatment of 500 consecutive cerebral aneurysms with long-term follow-up. AJNR Am J Neuroradiol 2013;34:2157-2162.

10. Jankowitz BT, Gross BA, Seshadhri S, Girdhar G, Jadhav A, Jovin TG, et al. Hemodynamic differences between Pipeline and coil-adjunctive intracranial stents. J Neurointerv Surg 2019; 11:908-911.

11. Koch MJ, Stapleton CJ, Raymond SB, Williams S, Leslie-Mazwi TM, Rabinov JD, et al. LVIS Blue as a low porosity stent and coil adjuvant. J Neurointerv Surg 2018;10:682-686.

12. Lawson MF, Newman WC, Chi YY, Mocco JD, Hoh BL. Stent-associated flow remodeling causes further occlusion of incompletely coiled aneurysms. Neurosurgery 2011;69:598-603; discussion 603-594.

13. Lim YC, Shin YS, Chung J. Flow Diversion via LVIS Blue Stent within Enterprise Stent in Patients with Vertebral Artery Dissecting Aneurysm. World Neurosurg 2018;117:203-207.

14. Matsuda Y, Chung J, Keigher K, Lopes D. A comparison between the new Low-profile Visualized Intraluminal Support (LVIS Blue) stent and the Flow Redirection Endoluminal Device (FRED) in bench-top and cadaver studies. J Neurointerv Surg 2018;10:274-278.

15. Nelson PK, Levy DI. Balloon-assisted coil embolization of widenecked aneurysms of the internal carotid artery: medium-term angiographic and clinical follow-up in 22 patients. AJNR Am J Neuroradiol 2001;22:19-26.

16. Poncyljusz W, Bilinski P, Safranow K, Baron J, Zbroszczyk M, Jaworski M, et al. The LVIS/LVIS Jr. stents in the treatment of wide-neck intracranial aneurysms: multicentre registry. J Neurointerv Surg 2015;7:524-529.

17. Wang J, Vargas J, Spiotta A, Chaudry I, Truner RD, Lena J, et al. Stent-assisted coiling of cerebral aneurysms: a single-center clinical and angiographic analysis. J Neurointerv Surg 2018;10: 687-692.

18. Zhang X, Zhong J, Gao H, Xu F, Bambakidis NC. Endovascular treatment of intracranial aneurysms with the LVIS device: a systematic review. J Neurointerv Surg 2017;9:553-557. 